EPJ manuscript No.

(will be inserted by the editor)

\title{
The electric form factor of the neutron and its chiral content
}

\author{
Murat M. Kaskulov and Peter Grabmayr \\ Physikalisches Institut, Universität Tübingen, D-72076 Tübingen, Germany
}

November 30, 2018

\begin{abstract}
Considering the nucleon as a system of confined valence quarks surrounded by pions we derive a Galster-like parameterization of the neutron electric form factor $G_{E}^{n}$. Furthermore, we show that the proposed parameterization can be linked to properties of the pion cloud. By this, the high quality data for the pion form factor can be used in predictions of $G_{E}^{n}$ in the low $Q^{2}$ region, where the direct double polarization measurements are not available.
\end{abstract}

PACS. 13.40.Gp Electromagnetic form factors - 12.39.Jh Nonrelativistic quark model

The electromagnetic (e.m.) form factors of the nucleons contain all the information on the internal nucleon structure and in particular they are very sensitive to the details of the interaction between the valence quarks. Already the first analyses by Hofstadter et al. 1] demonstrated that the proton electric form factor can be described by a dipole type form

$$
G_{D}\left(Q^{2}\right)=\left(1+Q^{2} / \Lambda^{2}\right)^{-2}
$$

Using the canonical $\Lambda_{D}^{2}=0.71 \mathrm{GeV}^{2}, G_{E}^{p}$ is reproduced up to four momentum transfer $Q^{2} \sim 1 \mathrm{GeV}^{2}$. Also the magnetic nucleon form factors, $G_{M}^{p}$ and $G_{M}^{n}$, are reproduced reasonably well. Recently, new precise polarization experiments and new analyses 2, 3, 4,5, 6 have brought clear evidence for the deviations of the form factors from the simple dipole form at high $Q^{2}$. Slight deviations from the dipole form at low $Q^{2}$ have been attributed to the underlying pionic and quarkonic structure of the nucleons 4 .

The neutron electric form factor $G_{E}^{n}$ is the most uncertain one due to its vanishing net charge and the absence of free neutron targets. The internal structure of the neutron is also reflected by a finite charge radius [7] 8 . Understanding the measured $\left\langle r^{2}\right\rangle_{n}=-0.115 \pm 0.003$ [9] is still an interesting goal, since the contributing Foldy term 8 of $3 F_{2}(0) / 2 M_{N}^{2}=-0.126 \mathrm{fm}^{2}$ almost equals the measured $\left\langle r^{2}\right\rangle_{n}$ value. At the same time, a recent analysis by Isgur 10] indicates that the Foldy term does not really explain the neutron charge radius and its charge distribution, because in leading order of the relativistic approximation to the constituent quark model (CQM) the Foldy term is canceled exactly by a contribution to the Dirac form factor $F_{1}$. This result was confirmed by Ref. 11. where it was argued that both, the success of CQM in reproducing the ratio of the proton to neutron magnetic moments and the success of the Foldy term in reproducing the observed charge radius of the neutron, are coincidental.
The extraction of the nonzero charge form factor $G_{E}^{n}$ from elastic electron scattering off the deuteron is rather model dependent 1,12,13. In recent years $G_{E}^{n}$ is being studied successfully by double polarization experiments 14 and the most recent parameterizations in the range up to $Q^{2} \sim 1 \mathrm{GeV}^{2}$ seem to converge [3,4,5],15]16]. The so called Galster [12] parameterization of $G_{E}^{n}$ is based on the same dipole form $G_{D}\left(Q^{2}\right)$ as for the proton. In oder to account for the condition $G_{E}^{n}\left(Q^{2}=0\right)=0$ required by the vanishing charge of the neutron, $G_{D}$ is multiplied by an appropriate function. Out of four trial functions [12,

$$
G_{E}^{n}\left(Q^{2}\right)=\frac{a_{G} \tau}{1+b_{G} \tau} G_{D}\left(Q^{2}\right)
$$

served best, where $\tau=Q^{2} / 4 M_{n}^{2}$ and $M_{n}=0.939 \mathrm{GeV}^{2}$ is the neutron mass. Originally 12, the parameter $a_{G}$ was set equal to $-\mu_{n}$ and the fit to the data resulted in $b_{G}=5.6$. More recent fits, e.g. Ref. 4, obtain $a \sim 1.73$ which reproduces the measured root mean square radius of the neutron and determines $b=4.62$.

Theoretically, up to now the Galster parameterization has no particular theoretical justification and is considered as a purely empirical description, i.e. $a_{G}$ and $b_{G}$, are fitting parameters. In this paper we wish to show that the parameterization of the neutron electric form factor $G_{E}^{n}$ can be derived directly under certain assumptions about the pionic content of the nucleon. We found that all parameters of Eq. (2) can be fixed by other experiments and are connected with the existence of pions in the nucleons. In order to disentangle shape and magnitude of $G_{E}^{n}$ we propose the use of the parameter $a^{\prime}$ which is defined as $a_{G}=a^{\prime} \cdot b_{G}$. The parameter $b_{G}$ can be related to the pion electromagnetic form factor $F_{\pi}$ and $a^{\prime}$ to the spectroscopic strength of the pions (number of pions) in the nucleon. With this prescription, reasonable parameters entering in Eq. (2) are obtained supporting the proposed interpretation. Using "our" Galster-like parameterization we are 
Murat M. Kaskulov, Peter Grabmayr: The electric form factor of the neutron and its chiral content

also able to relate the data for the pion e.m. form factor $F_{\pi}$ as provided by electroproduction experiments to the neutron electric form factor $G_{E}^{n}$. By this procedure, a high quality representation of $G_{E}^{n}$ can be obtained in the low momentum transfer region, where the direct double polarization data are still not available and would have large corrections due to final state interaction.

By now it is well established that the pion cloud plays an important role in understanding the variety of electromagnetic and hadronic properties of nucleons in the low-energy, non-perturbative region of QCD 17,18. The coupling of the pion field to the nucleon quark core and the resulting pion-loop (pion cloud) corrections are important ingredients of the so-called chiral quark models $(\chi \mathrm{QM})$ [18 where the pions are the Goldstone bosons generated by spontaneous breaking of $S U(2)_{R} \times S U(2)_{L}$ chiral symmetry. Easily the scheme can be generalized to the octet of light pseudo-scalar mesons $(\pi, \mathrm{K}, \eta)$ as provided by $S U(3)_{R} \times S U(3)_{L} 22$. Formally, the pion cloud can be introduced into the nucleon structure by the perturbative expansion of the nucleon Fock space. This procedure is reflected in the two-component structure of the nucleon wave function $\Psi_{N}=\left(\Psi_{3 q}, \Psi_{3 q+\pi}\right)$, where the first component, $\Psi_{3 q}$, represents the bare nucleon consisting of valence quarks, and the second one, $\Psi_{3 q+\pi}$, is the quark core dressed by a pion cloud which is mainly responsible for the soft physics. The presence of a soft pion cloud as an actual dynamical degree of freedom is crucial in understanding the neutron electric form factor $G_{E}^{n}$. In $\chi$ QM's at the one-pion loop level, the neutron charge form factor is a first order effect of the pion cloud and originates mainly from the Fock component of the neutron wave function consisting of a $\pi^{-}$cloud and a positively charged core of confined quarks. This physical picture gives a natural explanation of the nonvanishing charge distribution inside the neutron which otherwise, like in the simplest version of non-relativistic quark model (NRQM) with three valence quarks only, results in zero [17.

Consider the effective $S U(2)_{R} \times S U(2)_{L}$ Lagrangian of $\chi \mathrm{QM}$ which can be written formally as 18

$$
\mathcal{L}=\mathcal{L}_{\pi}^{(2)}+\mathcal{L}_{\pi q q}^{(1)}
$$

where the mesonic Lagrangian $\mathcal{L}_{\pi}^{(2)}$ of lowest-order in the derivative expansion is given by the nonlinear $\sigma$ model and the $\mathcal{L}_{\pi q q}^{(1)}$ is an effective pion-quark Lagrangian

$$
\mathcal{L}_{\pi q q}^{(1)}=-\frac{1}{4 f_{\pi}^{2}} \bar{\psi} \gamma^{\mu} \boldsymbol{\tau} \psi\left[\boldsymbol{\pi} \times \partial_{\mu} \boldsymbol{\pi}\right]-\frac{f_{\pi q q}}{m_{\pi}} \bar{\psi} \gamma^{\mu} \gamma_{5} \boldsymbol{\tau} \psi \partial_{\mu} \boldsymbol{\pi}
$$

where $\psi$ and $\boldsymbol{\pi}$ are a quark and pion fields, respectively, and the coupling constant $f_{\pi q q}$ characterizes the strength of the pion-quark interaction. The coupling of the pion field to the core of confined quarks, Eq. (4), results in pion-loop corrections as provided by the self-energy pionloop diagrams shown in Fig. 1] already on the hadronic level. Note that the underlying quark substructure of diagrams is reflected in appearance of hadronic form factors as shown by filled circles. The tadpole diagram (Fig. 10)

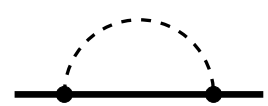

(a)

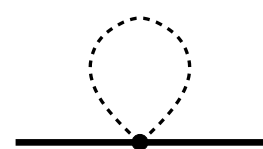

(b)
Fig. 1. One-pion loop self-energy diagrams. The intermediate state in (a) can be a nucleon or a $\Delta$. The tadpole (Hartree) diagram in (b) vanishes due to its isospin structure.

vanishes due to its isospin structure and Fig. 17) is characterized by the loop integral $-i \Sigma_{\alpha}(E)$ (see its explicite expression in Ref. [18), where the intermediate states $\alpha$ are a $N(939)$ or a $\Delta(1232)$. Fig. 17 introduces the pionic degrees of freedom into the nucleon structure and mathematically requires the renormalization of the total nucleon wave function $\left|\Psi_{N}\right\rangle^{R}=Z_{2}^{-1 / 2}\left|\Psi_{N}\right\rangle$, where the constant $Z_{2}$ is given by:

$$
Z_{2} \equiv 1-\sum_{\alpha} \partial \Sigma_{\alpha}(E) /\left.\partial E\right|_{E=M_{N}}
$$

and describes the probability of finding the "physical" nucleon in its bare three-quark valence Fock state. It also guarantees ${ }^{R}\left\langle\Psi_{N} \mid \Psi_{N}\right\rangle^{R}=1$. The value $Z_{2}$ can be related to the pionic content of the nucleon, namely to the pion spectroscopic factor, which represents the number of pions in the nucleon $S_{\pi}=\sum_{\alpha} S_{\pi}^{\alpha}=\left(Z_{2}-1\right) / Z_{2}$ or

$$
S_{\pi}^{\alpha}=-\left.\frac{1}{Z_{2}} \frac{\partial \Sigma_{\alpha}(E)}{\partial E}\right|_{E=M_{N}}
$$

The explicit introduction of the additional degrees of freedom in the nucleon structure will change its properties as compared to expectations based on simple quark models in which the nucleon is described as a system of three valence quarks only. For example, the coupling of the e.m. field $\mathcal{A}^{\mu}$ to the conserved e.m. currents due to a quark charge $\mathcal{Q}=\left[1 / 3+\tau_{3}\right] / 2$ which is achieved by a photon-quark interaction

$$
\mathcal{L}_{\gamma q q}^{(1)}=-e \mathcal{Q} \bar{\psi} \gamma^{\mu} \psi \mathcal{A}_{\mu}
$$

should be supplemented by the coupling of the photon field to pions and is given by the $\gamma \pi$ interaction Lagrangian

$$
\mathcal{L}_{\gamma \pi \pi}^{(2)}=-e\left[\boldsymbol{\pi} \times \partial^{\mu} \boldsymbol{\pi}\right]_{3} \mathcal{A}_{\mu}
$$

with additional contact interactions obtained by a gauge transformation of the pion field of Eq. (4) we get

$$
\begin{gathered}
\mathcal{L}_{\gamma \pi q q}^{(1)}=-e \frac{f_{\pi q q}}{m_{\pi}} \bar{\psi} \gamma^{\mu} \gamma_{5}[\boldsymbol{\tau} \times \boldsymbol{\pi}]_{3} \psi \mathcal{A}_{\mu}, \\
\mathcal{L}_{\gamma \pi \pi q q}^{(1)}=-e \frac{1}{4 f_{\pi}^{2}} \bar{\psi} \gamma^{\mu}[[\boldsymbol{\tau} \times \boldsymbol{\pi}] \times \boldsymbol{\pi}]_{3} \psi \mathcal{A}_{\mu} .
\end{gathered}
$$

Considering Eqs. (7 - 10) at the one-pion loop level, the full set of diagrams responsible for the neutron charge 


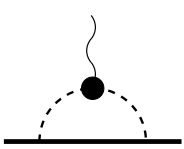

(a)

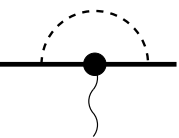

(d)

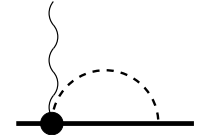

(b)

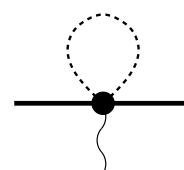

(e)

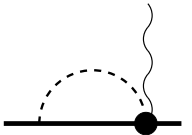

(c)

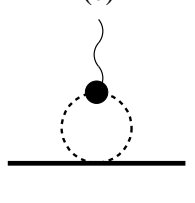

(f)
Fig. 2. E.m. currents at one-pion loop level.

form factor are shown in Fig. 2. Since the bare quark core, Eq. (7), and the "seagull" terms $\left(\sim \mathcal{O}\left(1 / M_{n}\right)\right)$ shown in Fig. 2] and 2r do not contribute and since the tadpole contact graphs, Fig. 2, and 2; cancel exactly, the leading order contribution arises from two one-pion loop processes with $\gamma q q$ (Fig. 21) and $\gamma \pi \pi$ couplings (Fig. 22 ), respectively. Following to the standard Feynmann rules the e.m. current operators responsible for Fig. 22a and 2/l can be constructed and in the Breit frame, where $Q^{2}=\boldsymbol{q}^{2}$, the resulting Sachs electric form factors of the neutron can be obtained as

$$
G_{E_{n}}^{\gamma q q}\left(\boldsymbol{q}^{2}\right)=\frac{\tilde{G}\left(\boldsymbol{q}^{2}\right)}{Z_{2}} \sum_{\alpha} \mathcal{K}_{N \alpha} \int \frac{d^{3} \boldsymbol{k}}{(2 \pi)^{3}} \boldsymbol{k}^{2} F^{2}\left(\boldsymbol{k}^{2}\right) \mathcal{C}_{\alpha}^{\gamma q q}(\boldsymbol{q}, \boldsymbol{k})
$$

$$
\begin{aligned}
G_{E_{n}}^{\gamma \pi \pi}\left(\boldsymbol{q}^{2}\right) & =-\frac{F_{\pi}\left(\boldsymbol{q}^{2}\right)}{Z_{2}} \sum_{\alpha} \mathcal{K}_{N \alpha} \\
& \times \int \frac{d^{3} \boldsymbol{k}}{(2 \pi)^{3}} F\left(\boldsymbol{k}^{2}\right) F\left(\boldsymbol{k}^{\prime 2}\right) \mathcal{C}_{\alpha}^{\gamma \pi \pi}\left(\boldsymbol{q}, \boldsymbol{k}, \boldsymbol{k}^{\prime}\right) \boldsymbol{k} \cdot \boldsymbol{k}^{\prime}
\end{aligned}
$$

where $\mathcal{K}_{N \alpha}=\left(f_{\pi q q} / m_{\pi}\right)^{2} C_{N \alpha}^{2} \chi^{\alpha}$ and $\boldsymbol{k}^{\prime}=\boldsymbol{k}+\boldsymbol{q}$. The coefficients are calculated algebraically within the CQM and result in $\chi^{N}=2, \chi^{\Delta}=-4 / 9, C_{N N}=5 / 3$ and $C_{N \Delta}=2 \sqrt{2} \cdot F_{\pi}(\boldsymbol{q})$ is the pion e.m. form factor and $F(\boldsymbol{k})$ is the nucleon axial form factor. Furthermore, $\tilde{G}\left(\boldsymbol{q}^{2}\right)$ in Eq. (11) represents the electric form factor of the quark core. At the one-pion loop level the e.m. current operators as provided by Fig. 2 involve a four dimensional integration over the momentum $k$ of the virtual pions. The momentum dependent factors $\mathcal{C}_{\alpha}^{\gamma \pi \pi}\left(\boldsymbol{q}, \boldsymbol{k}, \boldsymbol{k}^{\prime}\right)$ and $\mathcal{C}_{\alpha}^{\gamma q q}(\boldsymbol{q}, \boldsymbol{k})$ are the result of the integration over $k_{0}$, which we perform analytically by closing the contour in the lower half of the complex plane. Eqs. (11) and (12) are exact and hold for any chiral quark model involving a cloud of pions. Instead of doing model calculations at this stage we proceed further by simplifying them. It can be shown that in the limit of low $Q^{2}$, Eqs. (11) and (12) can be reduced to

$$
\left\{\begin{array}{c}
G_{E_{n}}^{\gamma q q}\left(\boldsymbol{q}^{2}\right) \\
G_{E_{n}}^{\gamma q q}\left(\boldsymbol{q}^{2}\right)
\end{array}\right\}=\left[\sum_{\alpha} \xi_{\alpha} S_{\pi}^{\alpha}\right]\left\{\begin{array}{c}
\tilde{G}\left(\boldsymbol{q}^{2}\right) \\
-F_{\pi}\left(\boldsymbol{q}^{2}\right) F\left(\boldsymbol{q}^{2}\right)
\end{array}\right\}
$$

where $S_{\pi}^{\alpha}$ is given by Eq. (6) and the spin-isospin factors are $\xi_{N}=2 / 3$ and $\xi_{\Delta}=-1 / 3$. Here the pion e.m. form factor $F_{\pi}(\boldsymbol{q})$, the nucleon axial form factor $F(\boldsymbol{q})$ and the core electric form factor $\tilde{G}\left(\boldsymbol{q}^{2}\right)$ are the relevant ingredients which define the $Q^{2}$ behavior of the reduced form factors. The latter two are characteristics of the quark core. Their appearance is quite unique in all the quark models - they do not exist in effective Lagrangian approaches at hadronic level. The important feature of the CQM, at least for our considerations, is that the axial $F\left(\boldsymbol{q}^{2}\right)$ and electric $\tilde{G}\left(\boldsymbol{q}^{2}\right)$ form factors have the same functional form: $F\left(\boldsymbol{q}^{2}\right)=\tilde{G}\left(\boldsymbol{q}^{2}\right)$. We use this fact to recombine Eqs. (13) as

$$
G_{E}^{n}\left(\boldsymbol{q}^{2}\right)=\tilde{G}\left(\boldsymbol{q}^{2}\right)\left[1-F_{\pi}\left(\boldsymbol{q}^{2}\right)\right] \sum_{\alpha} \xi_{\alpha} S_{\pi}^{\alpha}
$$

Furthermore, as known from electroproduction experiments the pion e.m. form factor $F_{\pi}\left(\boldsymbol{q}^{2}\right)$ can be fitted up to a few $\mathrm{GeV}$ [19,20] by a monopole term

$$
F_{\pi}\left(Q^{2}\right)=\Lambda_{\pi}^{2} /\left(\Lambda_{\pi}^{2}+Q^{2}\right)=\left(1+Q^{2} / \Lambda_{\pi}^{2}\right)^{-1},
$$

with a cut-off mass of $\Lambda_{\pi}^{2}=0.53 \mathrm{GeV}^{2}$ [19,20]. Using Eq. (15) the expression for $G_{E}^{n}$ can be written alternatively

$$
G_{E}^{n}\left(Q^{2}\right)=\sum_{\alpha} \xi_{\alpha} S_{\pi}^{\alpha} \cdot\left[\frac{Q^{2} / \Lambda_{\pi}^{2}}{1+Q^{2} / \Lambda_{\pi}^{2}}\right] \tilde{G}\left(Q^{2}\right)
$$

We define the two parameters $a^{\prime}$ and $b$ as

$$
b=4 M_{n}^{2} / \Lambda_{\pi}^{2} \quad \text { and } \quad a^{\prime}=\sum_{\alpha} \xi_{\alpha} S_{\pi}^{\alpha},
$$

If we assume in addition that the quark core electric form factor is given by the dipole ansatz, $\tilde{G}=G_{D}$, we then arrive at a Galster-like formulation for $G_{E}^{n}$ (cf. Eq. (2)

$$
G_{E}^{n}\left(Q^{2}\right)=a^{\prime} \frac{b \tau}{1+b \tau} G_{D}\left(Q^{2}\right) .
$$

where $\tau=Q^{2} / 4 M_{n}^{2}$. Already at this level "our" Galster form decouples the parameters responsible for shape $(b)$ and magnitude $\left(a^{\prime}\right)$, which reduces the uncertainty for $b$ by about a factor 2 in the fits. Note that the form of $G$ is model dependent; however, its generalization to other hadronic models is straightforward. Presently we have used the dipole form to make the direct correspondence with the Galster ansatz.

$a^{\prime}$ in Eqs. (17) and (18), which characterizes the spectroscopic strength of the pions in the nucleon, can be also fixed by imposing additional constraints coming from the experimental value of the neutron charge radius $\left\langle r^{2}\right\rangle_{n}$. The latter is defined as the slope of $G_{E}^{n}\left(Q^{2}\right)$ for $Q^{2} \rightarrow 0$, and applying this prescription to Eq. (16) we obtain for

$$
\left\langle r^{2}\right\rangle_{n}=-\left(6 / \Lambda_{\pi}^{2}\right) \sum \xi_{\alpha} S_{\pi}^{\alpha} \quad \text { or } \quad a^{\prime}=-\left\langle r^{2}\right\rangle_{n}\left(\Lambda_{\pi}^{2} / 6\right) .
$$

Assuming $\left\langle r^{2}\right\rangle_{n}$ and $F_{\pi}$ to be known, an even simpler expression for $G_{E}^{n}$ emerges

$$
G_{E}^{n}\left(Q^{2}\right)=-\frac{\left\langle r^{2}\right\rangle_{n}}{6} Q^{2} F_{\pi}\left(Q^{2}\right) G_{D}\left(Q^{2}\right)
$$




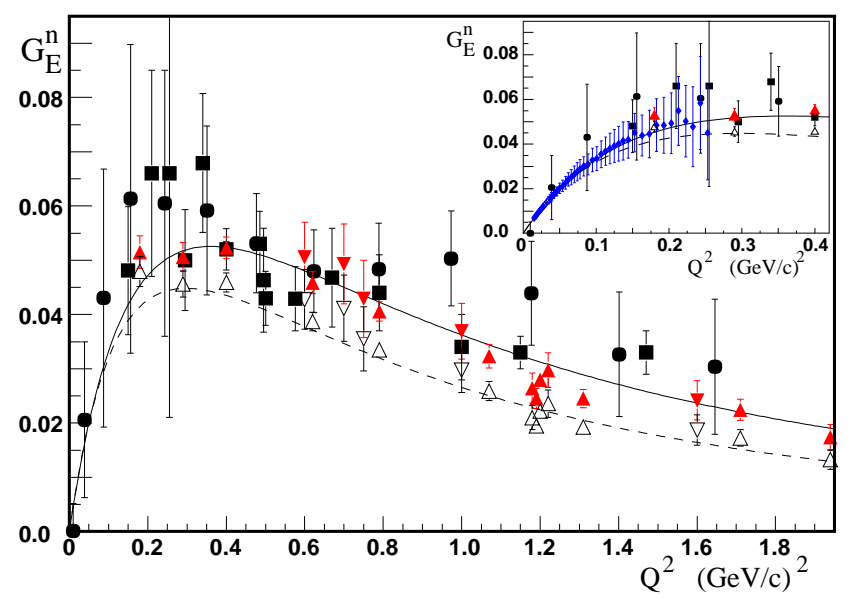

Fig. 3. The neutron electric $G_{E}^{n}$ form factor. Squares: polarization data from Refs. 14. Circles: elastic electron-deuteron data [16. Triangles: Eq. 20] with pion data of Refs. 19 20; (open symbols: $\Lambda^{2}=0.71 \mathrm{GeV}^{2}$, full: $\Lambda^{2}=0.86 \mathrm{GeV}^{2}$ ). The insert shows the low $Q^{2}$ range with additional pion data [21].

with no additional parameter. We mention that in Eq. (20) all quantities responsible for the properties of the pion cloud are experimentally accessible. This fact can be used to obtain the $G_{E}^{n}$ values directly from $\left\langle r^{2}\right\rangle_{n}$ and $F_{\pi}$.

Inserting the experimental values for $\left\langle r^{2}\right\rangle_{n}[9$ and for $F_{\pi}$ as extracted from electroproduction experiments 19, 20 directly into the above equation we obtain the data (open triangles) plotted in Fig. 3 For comparison, $G_{E}^{n}$ values selected by Ref. [4 are given by squares and the evaluation of Ref. 16 is presented by circles. The remarkable feature of the pion data is that they have smaller errors and that they lead to slightly smaller values for $G_{E}^{n}$ above $Q^{2}=0.3 \mathrm{GeV}^{2}$. In Fig. B the full curve represents the fit to the $G_{E}^{n}$ data 4,14 including the $\left\langle r^{2}\right\rangle_{n}$ value, whereas the dashed curve represents the fit to the pion data with $\Lambda_{D}^{2}=0.71 \mathrm{GeV}^{2}$ with reasonable $\chi^{2}$ (Table 1).

We note that, the use of $\Lambda_{D}^{2}=0.71 \mathrm{GeV}^{2}$ in Eq. (18) is not entirely correct as $\tilde{G}$ represents the core and not the extension of the total charge. Using parameters $a^{\prime}$ and $b$ according to the above formulae, a $\Lambda^{2}=0.86 \mathrm{GeV}^{2}$ is obtained from a fit to the polarization data (third fit of Table (1). The resulting curves from the first and third fit are identical below $Q^{2}=2 \mathrm{GeV}^{2}$ despite the different parameters. Using this $\Lambda^{2}=0.86 \mathrm{GeV}^{2}$, which corresponds to a $\sqrt{\left\langle r^{2}\right\rangle}=0.74 \mathrm{fm}$ of the core, results in derived $G_{E}^{n}$ values (full triangles in Fig. [3) similar to the measured polarization data. The insert show the obtained $G_{E}^{n}$ data for very low $Q^{2}$ range where more pion data 21] have been added.

Table 1. Galster fits to $G_{E}^{n}$ data. *) fixed in the fit.

\begin{tabular}{c|ccc|r}
\hline \hline ref. & $a^{\prime}$ & $b$ & $\Lambda^{2} / \mathrm{GeV}^{2}$ & $\chi^{2} /$ ndof \\
\hline 414 & $0.369 \pm 0.008$ & $4.69 \pm 0.11$ & $\left.0.71^{\star}\right)$ & $9.9 / 14$ \\
{$[19$} & $0.221 \pm 0.002$ & $8.21 \pm 0.16$ & $\left.0.71^{\star}\right)$ & $34.2 / 19$ \\
414 & $\left.0.26^{\star}\right)$ & $\left.6.65^{\star}\right)$ & $0.86 \pm 0.04$ & $9.7 / 15$ \\
\hline
\end{tabular}

In this momentum transfer region $F_{\pi}$ can be understood to provide a model independent input to $G_{E}^{n}$. Since $\tilde{G}$ is close to unity, $G_{E}^{n}$ is considered to be parameter free at low $Q^{2}$. Interestingly, the $G_{E}^{n}$ data from pion electroproduction fall right on top of the full curve. Clearly the pion data can only account for the soft contributions, however they give a lower limit for $G_{E}^{n}$ when using $\Lambda_{D}$. Parameter $a^{\prime}$ shows that the weighted sum of pion spectroscopic factors is in the range of $25 \%$ (Table 11).

In summary, in this work we have obtained a theoretical justification of the phenomenologically successful Galster parameterization of the neutron electric form factor $G_{E}^{n}$. We have shown that the chiral (pion cloud) content in the nucleon structure is the crucial ingredient that leads under some approximations to a Galster-like $Q^{2}$ dependence of $G_{E}^{n}$. We found that all parameters of Eq. (18), i.e. $a^{\prime}$ and $b$, can be fixed by other experiments and that they are connected with the existence of pions in the nucleons. The proposed parameterization of the $G_{E}^{n}$, Eqs. (18) and (20), decouples the parameters responsible for shape $(b)$ and magnitude $\left(a^{\prime}\right)$ and allows to derive precise $G_{E}^{n}$ data at low $Q^{2}$ where the direct measurements of $G_{E}^{n}$ by double polarization experiments require larger corrections factors due to the notorious final state interactions.

We finally mention, that an analog prescription, Eq. (20), can be set up for the strange electric form factors and the strange radius of the nucleon.

This work was supported by the Deutsche Forschungsgemeinschaft under contracts Gr1084/3 and GRK683, and LFSP(BW).

\section{References}

1. R. Hofstadter and R. Herman, Phys. Rev. Lett. 15 (1961) 293; E.E. Chambers and R. Hofstadter, Phys. Rev. 103 (1956) 1454.

2. E.J. Brash et al., Phys. Rev. C65 (2001) 051001(R).

3. J.J. Kelly, Phys. Rev. C66 (2002) 065203.

4. J. Friedrich and T. Walcher, Eur. Phys. J. A17 (2003) 607.

5. Haiyan Gao, Int. J. Mod. Phys. E12 (2003) 1.

6. M.M. Kaskulov and P. Grabmayr, Phys. Rev. C67 (2003) 042201(R).

7. E. Fermi and L. Marschall, Phys. Rev. 72 (1947) 1139.

8. L.L. Foldy, Phys. Rev. 87 (1952) 688; ibid 693.

9. S. Kopecky et al., Phys. Rev. C56 (1997) 2220.

10. N. Isgur, Phys. Rev. Lett. 83 (1999) 272.

11. D.B. Leinweber, A.W. Thomas and R.D. Young, Phys. Rev. Lett. 86 (2001) 5011.

12. S. Galster et al., Nucl. Phys. B32 (1971) 221.

13. S. Platchkov et al., Nucl. Phys. A570 (1990) 740.

14. $G_{E}^{n}$ data compiled in Table 1 of Ref. 4 .

15. P. Grabmayr and A.J. Buchmann, Phys. Rev. Lett. 86 (2001) 2237.

16. R. Schiavilla and I. Sick, Phys. Rev. C64 (2001) 041002.

17. G. A. Miller, Phys. Rev. C 66, 032201 (2002).

18. A.W. Thomas and W. Weise, The structure of the nucleon, Wiley-VCH, Berlin, 2001.

19. C.J. Bebek et al., Phys. Rev. 17 (1978) 1693.

20. J. Volmer et al., Phys. Rev. Lett. 86 (2001) 1713.

21. S.R. Amendolia et al., Nucl. Phys. B277 (1986) 168.

22. V. E. Lyubovitskij et al., Phys. Rev. C64, 065203 (2001). 